\title{
EVALUACIÓN DEL PLAN DE MANEJO DE ANIMALES DE CAZA EN LA RESERVA NACIONAL PUCACURO
}

\author{
Pedro PÉREZ-PEÑA ${ }^{1,2}$, Cristian GONZALES-TANCHIVA ${ }^{3}$, Marcial TRIGOSO-PINEDO² $^{2}$ \\ 1 Yavari: Conservación y Uso Sostenible (YAVACUS), Callao 702, Iquitos. E-mail: pedro.wildlife@gmail.com \\ 2 Programa de Investigación de la Biodiversidad Amazónica. Instituto de Investigaciones de la Amazonía Peruana (IIAP), \\ Av. Abelardo Quiñonez km 2.5, Iquitos. \\ 3 Reserva Nacional Pucacuro, Servicio Nacional de Áreas Naturales Protegidas por el Estado (SERNANP), Jorge Chávez \\ 930, Iquitos.
}

\section{RESUMEN}

La Reserva Nacional Pucacuro cuenta con un plan de manejo de animales de caza, entre los que se encuentran la huangana Tayassu pecari, el sajino Pecari tajacu, el venado rojo Mazama americana, el venado gris Mazama nemorivaga y el majas Cuniculus paca. Este plan busca conservar el ecosistema terrestre y ayudar en la economía familiar de los cazadores del pueblo indígena kichwa, mediante la venta de carne de monte a mejores precios en los mercados. Se estableció una cuota máxima anual de extracción de $300 \mathrm{~kg}$ de carne ahumada por cazador en la zona de caza, en función al consumo diario estimado de $1 \mathrm{~kg}$ de carne por persona. La caza de primates, tapires Tapirus terrestris y felinos fue prohibida, así como la de algunos edentados y reptiles protegidos por la legislación peruana. La colecta de información se realizó en el periodo que va del año 2011 al 2015, incluyendo la evaluación de la densidad poblacional de las especies, los registros de caza, entrevistas a cazadores y registros indirectos de las especies. Se evaluó la funcionalidad del plan de manejo usando cuatro modelos de sostenibilidad denominados vulnerabilidad o abundancia, esfuerzo, cosecha y consenso cultural. Tres de los modelos no evidenciaron sobrecaza, sin embargo, el modelo de abundancia evidenció poblaciones vulnerables a los cambios en las fluctuaciones del nivel del río. La implementación de planes de manejo de animales de caza por parte de las comunidades locales es una alternativa para la conservación de los bosques, ya que estos generan ingresos económicos y aseguran la reducción de algunas actividades que podrían dañar el ecosistema.

PALABRAS CLAVE: Manejo, cacería, carne de monte, comercio, Reserva Pucacuro.

\section{EVALUATING THE MANAgEMENT PLAN FOR GAME ANIMALS IN THE PUCACURO NATIONAL RESERVE}

\begin{abstract}
The Pucacuro National Reserve has a management plan for game animals such as the white-lipped peccary Tayassu pecari, collared peccary Pecari tajacu, red brocket deer Mazama americana, gray brocket deer Mazama nemorivaga and paca Cuniculus paca. This plan aims to conserve the terrestrial ecosystem and to increase the income of indigenous Kichwa families by selling managed bushmeat to more valuable markets. An annual maximum quota of $300 \mathrm{~kg}$ of smoked meat per hunter was established in the hunting area, according to the estimated daily consumption of $1 \mathrm{~kg}$ of meat per person. Hunting of primates, tapir Tapirus terrestris and cats was banned, along with those edentates and reptiles that are protected by Peruvian law. The information was collected from 2011 to 2015 and included the evaluation of the population density of game animals, hunting records, interviews with hunters and indirect records of the species. The functionality of the management plan was evaluated using four sustainable models named vulnerability or abundance, effort, harvesting and cultural consensus. These models generally do not show overhunting, however, the model of abundance indicated vulnerable populations by the influence of river level. The implementation of management plans for game animals is an alternative for the conservation of forests by local communities, this generates income and ensures the reduction of activities that could damage the ecosystem.
\end{abstract}

KEYWORDS: Management, hunting, bushmeat, business, Pucacuro Reserve. 


\section{INTRODUCCIÓN}

La carne de monte es un buen aporte de proteína para el poblador ribereño y su venta ayuda en la economía familiar, aunque su extracción excesiva o sobrecaza extingue localmente muchas especies dispersadoras de semillas y "arquitectas" de la estructura del bosque (Van Vliet, 2011; Altrichter et al., 2012; Harrison et al., 2013; Swamy et al., 2013). Este efecto es conocido como defaunación y tiene un efecto negativo en la conservación de la biodiversidad y el almacenamiento de carbono en el bosque (Bello et al., 2015; Osuri et al., 2016). Por lo tanto, el control de la sobrecaza mediante planes de manejo es muy importante para la conservación de los servicios ecosistémicos que brinda el bosque amazónico.

La implementación de planes de manejo de animales de caza es una alternativa importante para generar ingresos económicos en las comunidades locales. En la Amazonía peruana, como en otras regiones tropicales, la caza genera un gran comercio de carne de monte (Bendayán-Acosta et al., 2004; Davies \& Brown, 2007). En la ciudad de Iquitos, se comercializan anualmente más de 345 toneladas de carne de monte, incluyéndose en esta cifra reptiles, mamíferos y aves. En términos de volumen destacamos al sajino Pecari tajacu, al majas Cuniculus paca y a la huangana Tayassu pecari (Moya, 2011), como especies que son más comercializadas en los mercados locales. Sin embargo, la venta de carne de monte es informal y no hay incentivos para que los cazadores y comerciantes sean formales o legales. La informalidad puede deberse a los engorrosos y altos costos documentarios necesarios para formalizarse, pero quizá, la causa principal es la falta de motivación y proyección a largo plazo del productor/vendedor, que día a día, sobrevive ignorando que el mercado beneficia más a las personas visibles o formales (Ganoza-Durant \& Stiglich-Watson, 2015). El bajo precio de venta, ausencia de capital y la extracción insostenible, encasillan a la caza dentro de las actividades informales. Además, el actual marco legal no contempla el real uso del recurso, aspecto que dificulta a los decisores políticos conocer la magnitud del problema y encontrar sus posibles soluciones.

La cacería de animales silvestres fue débilmente normada desde los tiempos coloniales en los países con gran biodiversidad (Bowen-Jones, 2003), quizá porque se pensó que no contribuía al crecimiento económico del estado, como es el caso de las actividades mineras o de hidrocarburos (PulgarVidal et al., 2010). Estas actividades extractivas tuvieron su boom en la época dorada (2003-2013) debido a la gran demanda y subida de precios en el mercado internacional, sin embargo, tuvieron efectos socio-ambientales negativos sobre las poblaciones indígenas y ribereñas (Ganoza-Durant \& Stiglich-Watson, 2015).

La caza manejada sosteniblemente brinda alimento y salud a gran parte de la población, sobre todo a los sectores que menos se benefician de las políticas públicas, mejorando su economía familiar. El fomento de la caza sostenible de animales silvestres por parte de las comunidades locales es una alternativa de conservación de los bosques, generando ingresos y conservando animales dispersores de semillas como los grandes roedores, las aves y los primates. Asimismo, la caza sostenible reduce el riesgo de implementar otras actividades que podrían dañar el ecosistema.

Por lo tanto, es fundamental incentivar o motivar a los pobladores locales para que usen sosteniblemente sus recursos, realizar la caza de manera formal y así ser visibles en un mercado que permita brindar mayores beneficios económicos a largo plazo. La caza también puede verse influenciada por factores naturales como las prolongadas crecientes de los ríos, que afectan a las poblaciones de animales terrestres en zonas estacionalmente inundables (Bodmer et al., 2014).

En este contexto, el año 2014, la Reserva Nacional Pucacuro (RNP) aprobó un plan de manejo de huangana Tayassu pecari, sajino Pecari tajacu, venado rojo Mazama americana, venado gris Mazama nemorivaga y majas Cuniculus paca, con fines comerciales. Estas especies fueron seleccionadas debido a que son menos vulnerables a la sobrecaza (Bodmer et al., 1997). El plan de manejo busca conservar los bosques de la cuenca del río Pucacuro y ayudar en la economía familiar de los pobladores kichwas mediante la venta de carne de monte a mejores mercados, bajo estándares de uso sostenible. En base a las reuniones comunales consensuadas y al consenso del uso de $1 \mathrm{~kg} /$ día de carne ahumada por familia y conociendo que los pobladores tienen otras fuentes proteicas cómo animales de corral y peces, se estableció una cuota de $300 \mathrm{~kg} /$ año por cazador. Este plan estableció una cuota máxima de extracción de $100 \mathrm{~kg}$ de carne ahumada por cazador, tres veces al año, realizada sólo en la zona de caza de la reserva. Esta cuota es menor a los reportes de extracción desordenada que se realizaban antes en la cuenca (Soini et al., 2001). Además, se prohibió la caza de primates, del tapir Tapirus terrestres y felinos, así como al de algunos edentados y reptiles vedados por la legislación nacional (SERNANP, 2014). 
Teniendo en consideración que la base de todo comercio de recursos naturales debe realizarse bajo estándares de uso sostenible, el presente trabajo evaluó la funcionalidad del plan de manejo de la RNP con fines comerciales. Utilizamos cuatro modelos de sostenibilidad (abundancia, esfuerzo, cosecha, consenso cultural) para estimar el efecto de la caza sobre las poblaciones de animales. Asimismo, el modelo de abundancia, consideró cambios en el nivel del río sobre las estimaciones poblacionales de animales de caza. De esta forma, se espera ayudar a regularizar el mercado de carne de monte y al mismo tiempo dar resultados confiables sobre una alternativa de conservación por los pobladores locales a través del uso sostenible de la fauna silvestre, y así apoyar a la toma de decisiones y la mejora de la legislación nacional.

\section{MATERIAL Y MÉTODOS}

\section{ÁREADE ESTUDIO}

El estudio se desarrolló en la Reserva Nacional Pucacuro, ubicada al norte del departamento de Loreto. En el 2010, se designó una zona de caza dentro de la reserva que abarca un área total de 155,209 ha (Figura 1), área suficientemente grande para realizar el manejo de la caza de especies de animales silvestres con alta densidad y tasa de extracción, como la huangana Tayassu pecari (SERNANP, 2013 y 2014). La selección de un gran área fue priorizada para asegurar el uso sostenible del recurso y así evitar la erosión genética de las especies (Biondo et al., 2011),

El área alberga hábitats inundables y de tierra firme. En los hábitats inundables se encuentran los bosques de terraza baja y aguajales, en los de tierra firme están los bosques de terraza media, alta y colina baja. Geológicamente, la zona de caza se localiza sobre las formaciones Pebas y Nauta, la primera incluye aquellos bosques con suelos arcillosos muy ricos en nutrientes y contiene a casi todas las colpas de la zona, mientras la formación Nauta alberga bosques con suelos arcillosos muy pobres (Higgins et al., 2011). La cuenca alta del Río Pucacuro alberga mayormente bosques de tierra firme sobre Formación Pebas, mientras que la cuenca media y baja, albergan bosques de tierra firme e inundable sobre la formación Nauta.

\section{COLECTADEDATOS}

\section{Transectos en banda}

Las evaluaciones se iniciaron el año 2012, un año después de establecerse la cuota de caza y tres años antes de aprobarse el plan de manejo con fines comerciales. El transecto en banda es uno de los métodos más usados en los estudios de fauna silvestre (Burnham et al., 1980). Durante el estudio, se abrieron tres transectos en cada una de las cuatro zonas de muestreo, que fueron evaluados entre las 7:00 y las 15:00 h, desde setiembre del 2012 hasta noviembre del 2015, durante las épocas de creciente y vaciante. Cada evaluación de campo duró al menos cuatro semanas, con tres o cuatro personas realizando las evaluaciones simultáneamente. En cada avistamiento se anotaron informaciones sobre el hábitat, distancia en el transecto, hora de registro, distancia del recorrido y clima, entre otras observaciones. Los transectos fueron establecidos con un diseño sistemático, evitando zonas impenetrables como aguajales puros o cochas inundadas. Los transectos tuvieron orientación radial para conseguir independencia de las réplicas. El ancho de banda o ancho fijo de la huangana $T$. pecari fue de $30 \mathrm{~m}$, del sajino $P$. tajacu y venados $M$. americana y M. nemorivaga fueron de $20 \mathrm{~m}$. En el caso de primates se usó ancho fijo de $25 \mathrm{~m}$ en Pithecia aequatorialis, $20 \mathrm{~m}$ en Lagothrix poeppigii y Saimiri macrodon, y $15 \mathrm{~m}$ en Leontocebus lagonotus y Callicebus discolor.

\section{Conteo de madrigueras}

Se buscaron madrigueras activas a lo largo de los transectos establecidos en una franja de $5 \mathrm{~m}$ a ambos lados del transecto. El cálculo de la densidad del majás Cuniculus paca se realizó en base al número de madrigueras activas divididas por 3.5 (Beck King et al., 1999).

\section{Registros de caza}

Al final de cada actividad de caza, los cazadores registraron la carne de monte en una ficha llamada registro de caza, que fue llenada con ayuda de los guardaparques de los puestos de control de la reserva. Esta información se colectó del 2011 al 2015, e incluye las cantidades extraídas, lugares, esfuerzo de caza, sexo y estadío (a simple vista) de las especies. La unidad de muestreo fue el grupo de cazadores, generalmente compuesto por tres o cuatro personas.

\section{Entrevistas de consenso cultural}

Durante el 2012, 2015 y 2016 se entrevistaron a cazadores de la comunidad indígena Kichwa 28 de Julio, usando el Modelo de Consenso Cultural (Weller, 2007). Seguimos las consideraciones de Van Holt et al. (2010) y Perez-Peña et al. (2012) para la evaluación de sus percepciones sobre la abundancia de animales de caza. 


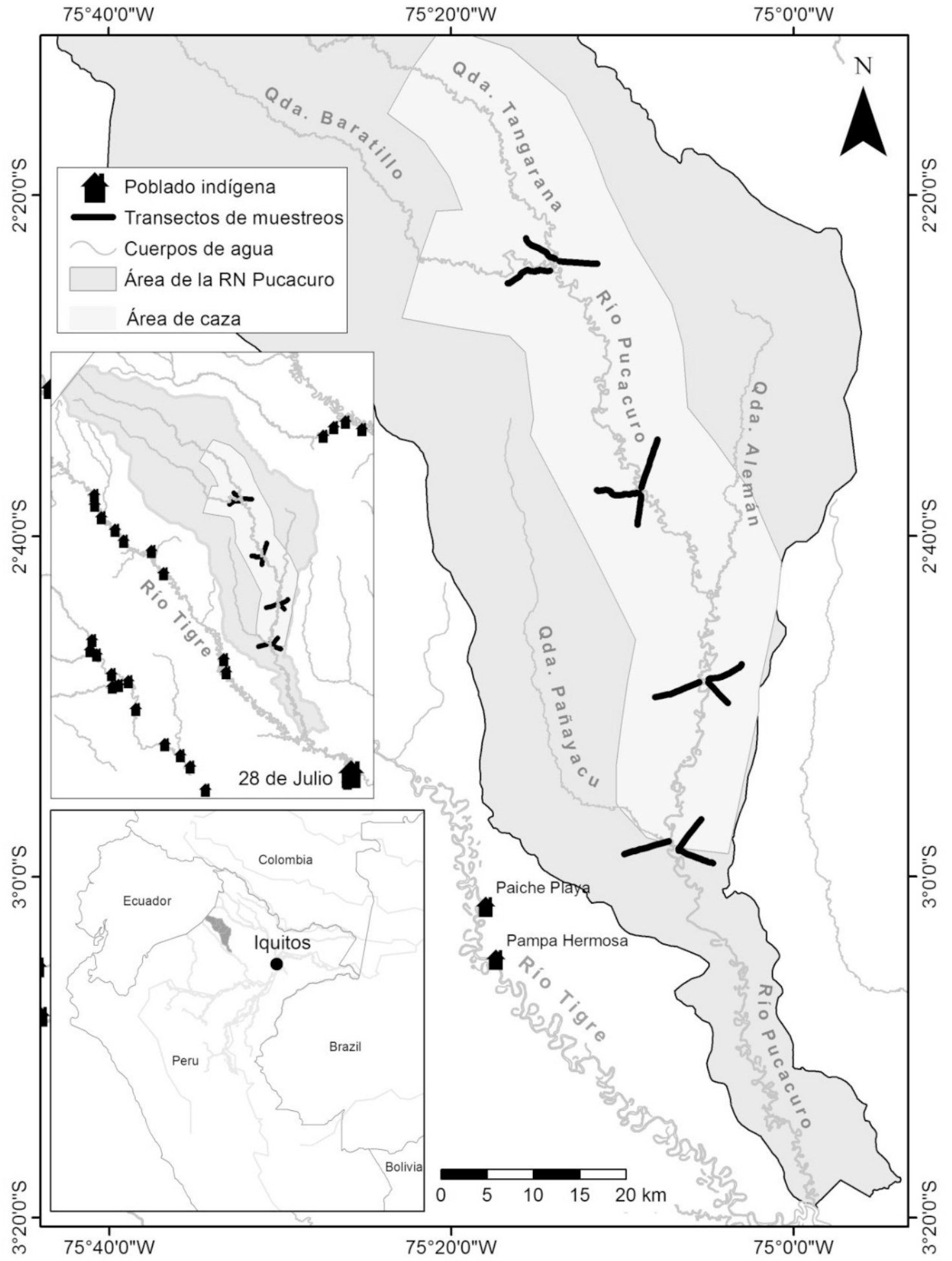

Figura 1. Mapa de ubicación de la zona de estudio mostrando los transectos de evaluación de animales de caza y la comunidad indígena Kichwa 28 de Julio, Loreto, Perú. 
La entrevista consistió en presentar imágenes laminadas a los cazadores y preguntar cuáles de aquellos animales eran escasos y cuáles eran abundantes. Las imágenes que no fueron seleccionadas se consideraron como frecuentes.

\section{Area de ocupación}

El área de ocupación es el área dentro de la extensión de presencia que es ocupada por una especie. Esta medida se enfoca al cálculo exacto del área que está ocupando una especie. Para calcular esta área se usó el rango domiciliario estimado en 20 $\mathrm{km}^{2}$ (Keuroghlian et al., 2004), como escala apropiada para identificar un grupo independiente de la huangana $T$. pecari.

\section{Nivel del río}

En la desembocadura del río Pucacuro se instalaron barras de medida, aque ayudaron a medir diariamente el nivel del río desde noviembre del 2010 hasta noviembre del 2012.

Los niveles del río Amazonas en la ciudad de Iquitos desde el 2011 al 2015, se obtuvieron gracias a la información colectada por el Servicio de Hidrografía de la Marina de Guerra del Perú. El nivel de inundación crítica del río Amazonas a la altura de la ciudad de Iquitos, se obtuvo de la información del caudal máximo de inundación del SENAMHI.

\section{ANÁLISIS DE DATOS}

\section{Cálculo de la densidad}

Se usó el método de ancho fijo para el cálculo de la densidad (Burnham et al., 1980), en el caso de majas $C$. paca se usó su adaptación que es: $D=(n)$ 3.5) / ( $L W 2$ ), donde $n$ es el número de madrigueras, $L$ es la longitud del transecto y $W$ es el ancho de búsqueda y " 2 " es la constante que indica la búsqueda a ambos lados (Beck King et al., 1999).

\section{Modelo de abundancia}

Este modelo evalúa las diferencias de las abundancias en los periodos de evaluación. Se considera que las reducciones en abundancia son sinónimo de vulnerabilidad, es decir, las especies que reducen sus poblaciones de una evaluación a otra, se consideran vulnerables a la caza.

Para determinar los cambios entre los años de monitoreo se usó una prueba ANOVA de una vía para muestras repetidas y prueba post-hoc LSD. Se usó índices de abundancia de tres localidades durante la temporada de vaciante, con la finalidad de homogenizar el espacio y la temporada de todos los años.

\section{Modelo de esfuerzo}

Los registros de caza se analizaron mediante el índice de Captura Por Unidad de Esfuerzo (CPUE) el cual asume que, una disminución del CPUE indica sobrecaza, mientras que un aumento o estabilidad refleja incremento o estabilidad poblacional (Bodmer \& Robinson, 2004). Se usaron días de caza como unidades de esfuerzo y las comparaciones se realizaron con la prueba Kruskal Wallis, que asume muestras independientes de variables que no siguen una distribución normal.

\section{Modelo de cosecha}

Este modelo evalúa si la caza es sostenible mediante la comparación de la cosecha y la producción. La fórmula para calcular la productividad es $\mathrm{P}=(0.5 \mathrm{D})(\mathrm{Yg})$, donde $\mathrm{Y}$ es la productividad reproductiva bruta, $\mathrm{g}$ es el número de gestaciones por año y $\mathrm{D}$ es la densidad poblacional (es considerado sólo la mitad asumiendo un sex ratio de $50 \%$ de hembras). La densidad se calculó usando información de época de creciente y vaciante. La información de productividad bruta de la huangana, sajino, venado y majas provino de estudios realizados por Mayor et al. $(2009,2010,2011)$ y Aquino et al. (2009), respectivamente; la información de número de gestaciones de las cuatro especies se obtuvo de Aquino et al. (2001). La cosecha o presión de caza se obtiene dividiendo el número de individuos cazados y el área de caza y la sostenibilidad se obtiene dividiendo la presión de caza y la productividad. De acuerdo a Bodmer \& Robinson (2004), el límite máximo de la cosecha sostenible es $50 \%$ para el majás y $40 \%$ para el sajino, huangana y venados.

\section{Modelo de consenso cultural}

El análisis de consenso cultural siguió al modelo formal, el cual asume consenso cuando las respuestas son unidimensionales o hay un sólo patrón de respuestas. Para esto, usamos un análisis multivariado que busca entender los principales ejes de variación en las respuestas de las diferentes personas. Entonces, consideramos que si la proporción entre el primer y segundo eigenvalue $o$ autovalor, es mayor a tres, indica que sólo una dimensión está explicando dos tercios de la variabilidad de la muestra, en consecuencia,las entrevistas se ajustan al modelo unidimesional o de un solo patrón de respuestas. El modelo proporcionó los valores de abundancia como los valores promedios de respuesta de los informantes. Estos análisis se realizaron con el programa UCINET 6.45 (Borgatti et al., 2002). Las comparaciones entre los 
años de entrevistas fueron realizadas usando la prueba no paramétrica de Kruskal Wallis, la cual asume tres o más muestras independientes de datos ordinales o discretos. Los cálculos se realizaron con el programa Bioestat 5.0 (Ayres et al., 2007).

\section{RESULTADOS}

\section{MODELODE ABUNDANCIA}

Los índices de abundancia durante la temporada de vaciante del sajino Pecari tajacu, huangana Tayassu pecari, venado rojo Mazama americana y venado gris Mazama nemorivaga, parecen haber disminuido gradualmente a lo largo de los cuatro años de evaluación. De estas especies, sólo T. pecari y $M$. americana llegaron a estar ausentes en el último censo en las localidades de Serna, Posayo y Pañayacu; estuvieron presentes aguas arriba, en la localidad de Ishkay Yacu, donde hay menos bosque inundable. Una disminución en el índice de abundancia indicaría que estas especies, durante la temporada de vaciante, fueron susceptibles a la sobrecaza, pero parece que esta disminución no está relacionada a la caza sino a factores climáticos. Es así que algunos primates, a pesar de estar prohibida su caza, muestran similar patrón de disminución en la abundancia a través del tiempo, pero sin llegar a registros de poblaciones totalmente ausentes, porque son especies arborícolas (Figura 2).

La disminución poblacional fue marcadamente significativa para los monos medianos, como el mono negro Sapajus macrocephalus y huapo negro Pithecia aequatorialis $\left(\mathrm{F}_{3.24}=3.33, \quad \mathrm{p}<0.05 \mathrm{y}\right.$ $\mathrm{F}_{3,24}=4.50, \mathrm{P}<0.05$, respectivamente). Sin embargo, los monos grandes como el mono choro Lagothrix lagotricha poeppigii y las especies pequeñas como el tocón colorado Callicebus discolor, el pichico común Leontocebus lagonotus y fraile Samiri macrodon, no mostraron disminución significativa de sus poblaciones.

El factor natural que estaría involucrado en esta disminución, es el nivel del río, muy variable durante los últimos años. Los niveles del río Pucacuro están relacionados con los del río Amazonas ( $\mathrm{rs}=0.68$, $\mathrm{P}<0.0001$ ). Entre el 2011 y 2015, los niveles medios del río Amazonas se incrementaron gradualmente; la duración de la inundación crítica fue de 74 días en el 2011 y 180 días en el 2015, es decir, el bosque permaneció más tiempo inundando en los últimos cuatro años.

Registros directos e indirectos durante todo el año de la huangana $T$. pecari, indicaron que el área usada disminuyó desde el 2013 al 2015, aunque se incrementó ligeramente durante el último año, pero aún no fue igual a la reportada en 2013 (Figura 4). La diferencia del área de ocupación del 2013 y 2015 es de cuatro rangos domiciliarios $\left(80 \mathrm{~km}^{2}\right)$ registrados en la parte alta de la zona de caza, aunque durante el 2015 se registraron en lugares de la parte baja que no fueron registrados en el 2013. El 2014 fue al año con menor área de ocupación $\left(380 \mathrm{~km}^{2}\right)$.

\section{MODELO DE ESFUERZO}

La especie más cazada fue la huangana T. pecari con 2178 individuos extraídos. Su nivel de extracción estuvo relacionado con los niveles del río y el tiempo de inundación crítica. El año 2011 tuvo mayor extracción (567 individuos) y tuvo el nivel medio más bajo (112.78 m.s.n.m) y sólo 74 días de inundación crítica, mientras que durante el 2014 y 2015 tuvieron la menor cantidad extraída (675 individuos $=21 \%$ ) pero con los niveles medio del río más altos (114.75 y 115.42 m.s.n.m., respectivamente) y 113 y 180 días de inundación crítica, respectivamente.

No obstante, los análisis de la tasa de extracción, que incluye el esfuerzo de caza, indicaron que no hubo cambio del CPUE a través del tiempo, en el sajino P. tajacu $(\mathrm{H}=4.08, \mathrm{P}=0.395)$, majas $C$. paca $(\mathrm{H}=4.76, \mathrm{P}=0.312)$ y venado rojo $M$. americana $(\mathrm{H}=5.252, \mathrm{P}=0.262)$, pero si mostró cambios significativos en la huangana T. pecari $(\mathrm{H}=11.04$, $\mathrm{P}=0.026$ ), especialmente durante el 2014 que disminuyó significativamente, después de dos años de constantes crecientes prolongadas de aproximadamente cinco meses y con el nivel medio del río que seguía subiendo. Durante el 2015 se estimó una ligera recuperación del CPUE el cual se relaciona con el menor tiempo de inundación crítica del año anterior, 2014 (Figura 5).

\section{MODELO DE COSECHA}

La cacería de las cuatro especies fue realizada debajo de los límites de sostenibilidad (Tabla 1). Durante los cuatro años, el rango del porcentaje de producción cosechada de la huagana T. pecari fue de 7.48 a 26.67 , en el sajino $P$. tajacu, fue de 2.49 a 10.26 , en el venado rojo $M$. americana fue de 3.45 a 14.48 y en el majas $C$. paca fue de 0.53 a 0.71 . Estos resultados mostraron que la extracción de los animales está muy por debajo de los límites máximos sostenibles (MSY). Es importante mencionar que el incremento del nivel del río y el mayor tiempo de inundación pueden jugar un rol importante en la regulación de la extracción, en especial de la huangana $T$. pecari y majas $C$. paca.

\section{MODELO DE CONSENSO CULTURAL}

Las entrevistas realizadas a los cazadores 
durante los tres años, tuvieron un patrón unidimensional de respuestas, es decir, hubo consenso en las respuestas (Eigenvalue $>3$ ). El nivel de acuerdo entre cazadores pareció que fue ligeramente mayor en el último año, cuando hubo un número menor de entrevistados (Tabla 2). Los resultados indicaron que los cazadores percibieron en consenso que las especies más abundantes fueron la huangana Tayassu pecari, majas Cuniculus paca, y sajino Pecari tajacu y las especies frecuentes fueron el venado rojo Mazama americana y venado gris Mazama nemorivaga. De las tres especies abundantes sólo $C$. paca incrementó su percepción de abundancia $(\mathrm{P}<0.05)$, mientras que de las especies consideradas frecuentes sólo M. americana pasó de la categoría frecuente a abundante durante el último año, aunque la diferencia en la percepción no resultó significativa.

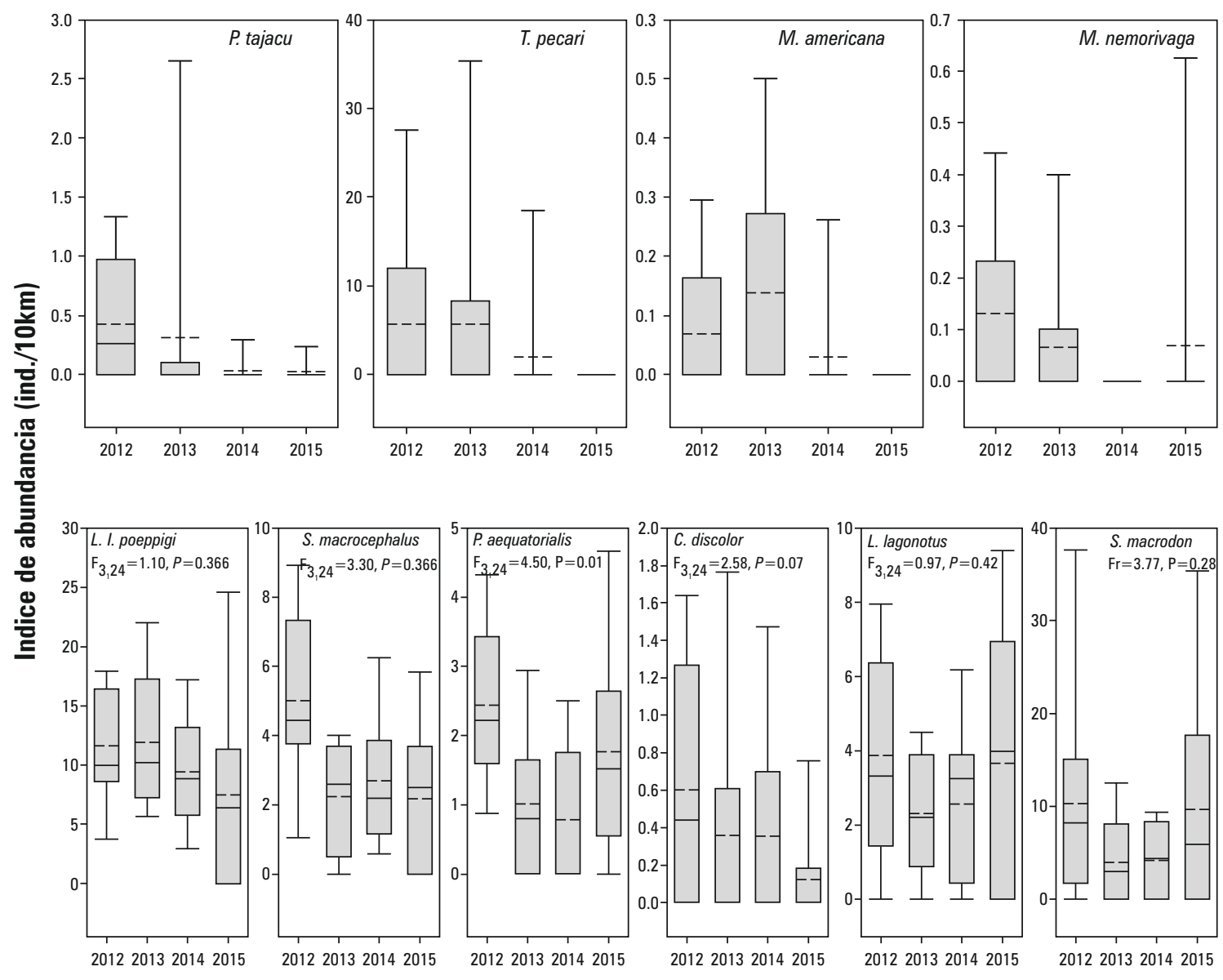

Figura 2. Monitoreo de la abundancia de cuatro especies de caza y primates usando el índice de abundancia durante la época de vaciante del río. La caja indica el 25 y $75 \%$ de cuartiles ( $50 \%$ de la muestra), la línea continua media es la mediana, la línea media cortada es la media. Los círculos negros son los valores extremos. 

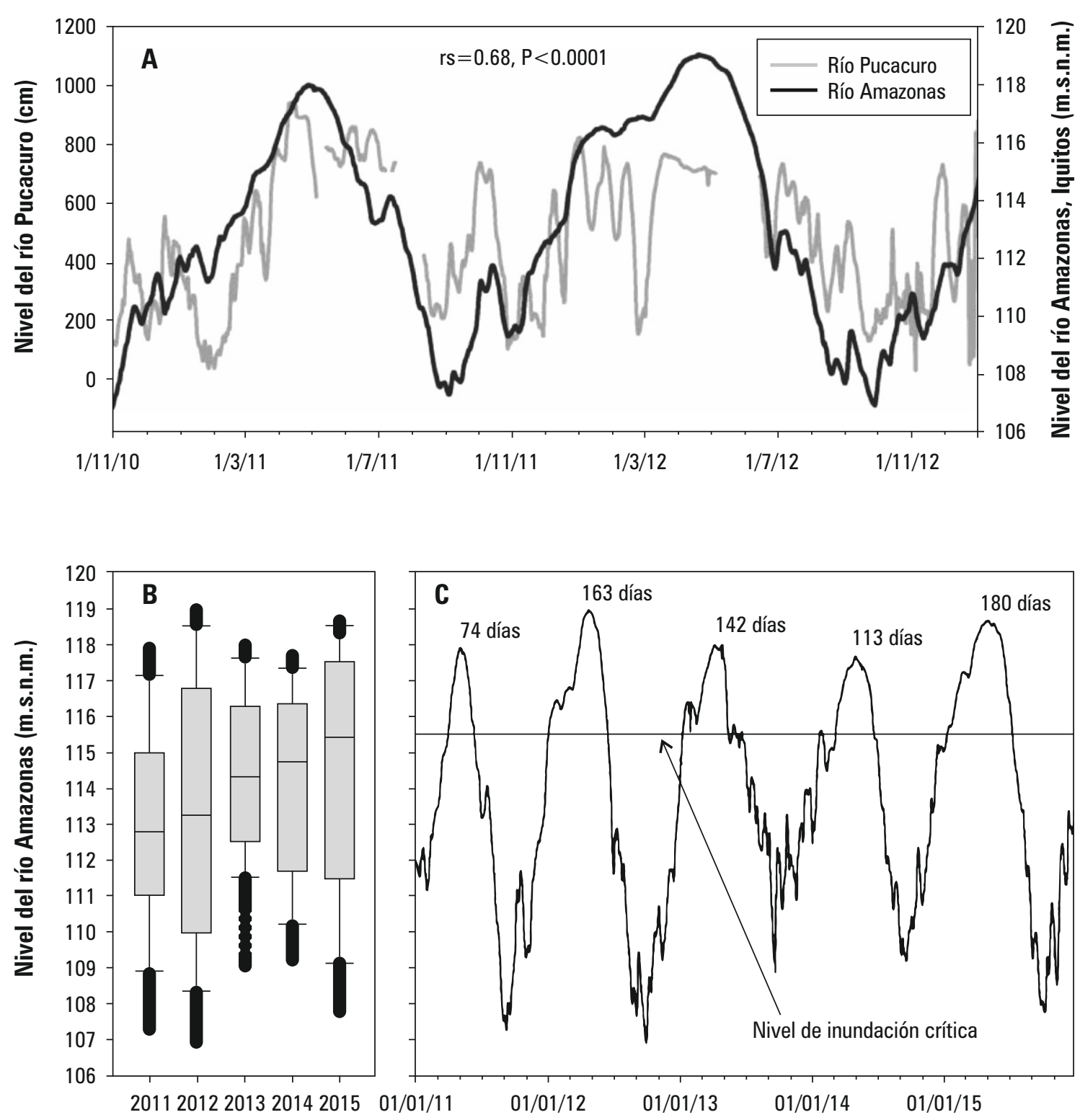

Figura 3. Comportamiento de los niveles de río. A) Relación del río Pucacuro y Amazonas a la altura de la ciudad de lquitos, B) Niveles medio del río Amazonas durante los últimos cinco años, C) Niveles críticos de inundación del río Amazonas asumiendo el punto crítico de 115.5 m.s.n.m en base al caudal máximo crítico. 

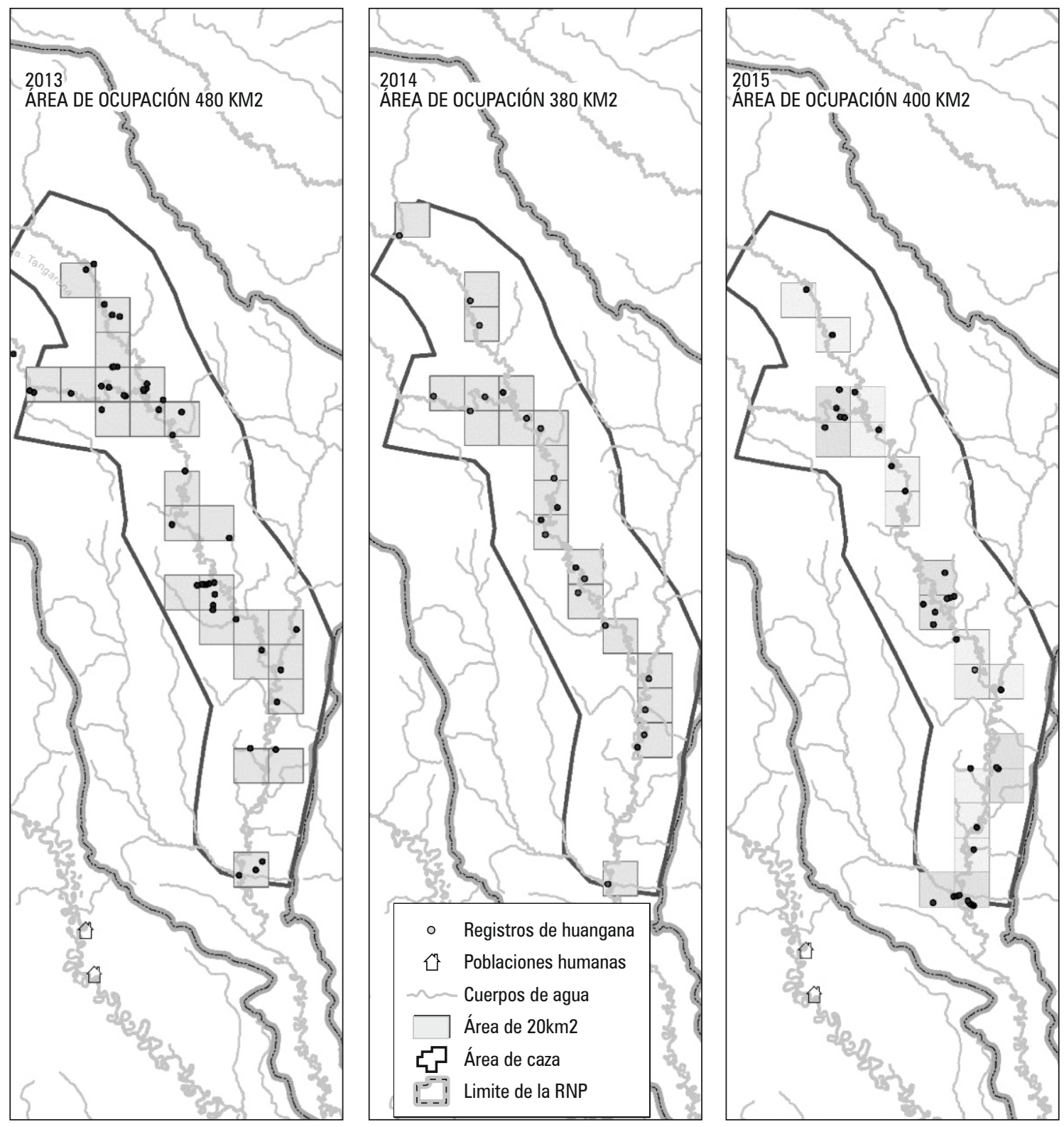

Figura 4. Área de ocupación de la huangana T. pecari en la zona de caza, durante los últimos tres años. 


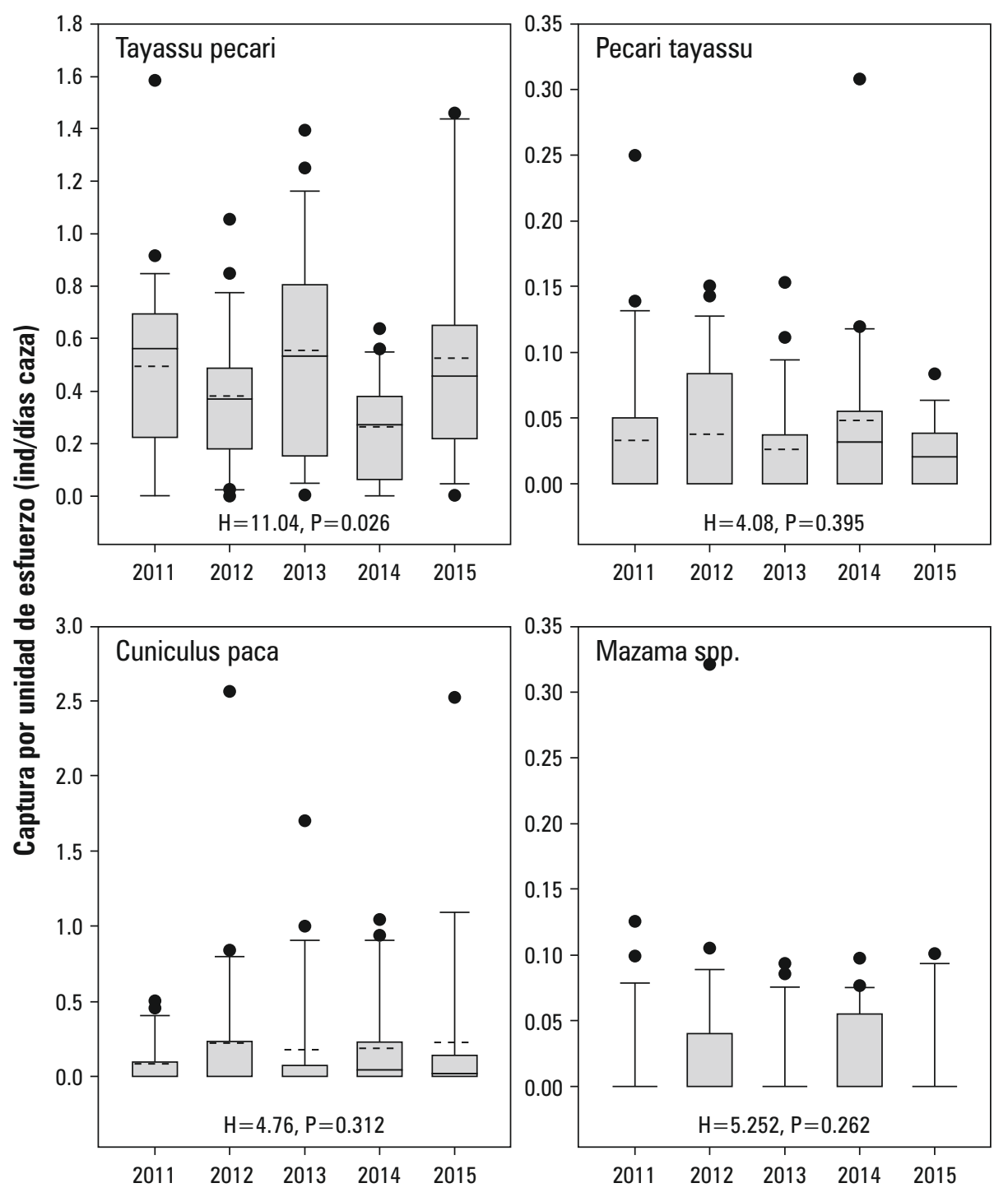

Figura 5. Monitoreo de las cuatro especies más cazadas mediante el modelo de esfuerzo usando la Captura por Unidad de Esfuerzo (CPUE) y los resultados de la prueba de significancia. 
Tabla 1. Monitoreo de la sostenibilidad de la caza mediante el modelo de cosecha de las cuatro especies más cazadas en la zona de caza de la Reserva Nacional Pucacuro.

\begin{tabular}{|c|c|c|c|c|c|c|c|c|c|c|c|c|c|c|}
\hline \multirow[t]{2}{*}{ Especie } & \multicolumn{4}{|c|}{ Tayassu pecari } & \multicolumn{4}{|c|}{ Pecari tajacu } & \multicolumn{4}{|c|}{ Mazama americana } & \multicolumn{2}{|c|}{$\begin{array}{c}\text { Cuniculus } \\
\text { paca }\end{array}$} \\
\hline & 2012 & 2013 & 2014 & 2015 & 2012 & 2013 & 2014 & 2015 & 2012 & 2013 & 2014 & 2015 & 2014 & 2015 \\
\hline $\begin{array}{l}\text { Individuos } \\
\text { extraídos/año }\end{array}$ & 400 & 536 & 305 & 370 & 37 & 27 & 42 & 16 & 11 & 7 & 10 & 4 & 135 & 98 \\
\hline $\begin{array}{l}\text { Área de caza } \\
(\mathrm{km} 2)\end{array}$ & 1552 & 1552 & 1552 & 1552 & 1552 & 1552 & 1552 & 1552 & 1552 & 1552 & 1552 & 1552 & 1552 & 1552 \\
\hline $\begin{array}{l}\text { Presión anual de } \\
\text { caza (ind./km2) }\end{array}$ & 0.26 & 0.35 & 0.2 & 0.24 & 0.02 & 0.02 & 0.03 & 0.01 & 0.01 & 0.005 & 0.006 & 0.003 & 0.087 & 0.06 \\
\hline $\begin{array}{l}\text { Productividad } \\
\text { bruta }\end{array}$ & 0.62 & 0.62 & 0.62 & 0.62 & 0.61 & 0.61 & 0.61 & 0.61 & 0.48 & 0.48 & 0.48 & 0.48 & 0.79 & 0.79 \\
\hline $\begin{array}{l}\text { Gestaciones } \\
\text { al año }\end{array}$ & 1.69 & 1.69 & 1.69 & 1.69 & 1.88 & 1.88 & 1.88 & 1.88 & 1.7 & 1.7 & 1.7 & 1.7 & 2 & 2 \\
\hline $\begin{array}{l}1 / 2 \text { densidad } \\
\text { (ind./km2) }\end{array}$ & 3.29 & 1.92 & 0.72 & 1.46 & 0.76 & 0.61 & 0.23 & 0.23 & 0.06 & 0.16 & 0.17 & 0.08 & 7.76 & 7.48 \\
\hline $\begin{array}{l}\text { Productividad } \\
\text { anual }\end{array}$ & 3.45 & 2.01 & 0.75 & 1.53 & 0.87 & 0.7 & 0.26 & 0.26 & 0.05 & 0.13 & 0.14 & 0.07 & 5.1 & 3.02 \\
\hline $\begin{array}{l}\text { \% Producción } \\
\text { cosechada }\end{array}$ & 7.48 & 17.17 & 26.67 & 15.69 & 2.74 & 2.49 & 10.26 & 3.91 & 14.48 & 3.45 & 4.64 & 3.95 & 0.71 & 0.53 \\
\hline Sobrecaza & No & No & No & No & No & No & No & No & No & No & No & No & No & No \\
\hline
\end{tabular}

Tabla 2. Monitoreo de la abundancia de las cuatro especies más cazadas en la zona de caza de la Reserva Nacional Pucacuro usando el modelo de Consenso Cultural.

\begin{tabular}{l|ccc|c|}
\hline \multirow{2}{*}{ Especie } & \multicolumn{3}{c}{ Score (raro a abundante:1 a 3) } & \multirow{2}{*}{ Kruskal-Wallis } \\
\cline { 2 - 4 } & $\mathbf{2 0 1 2}$ & $\mathbf{2 0 1 5}$ & $\mathbf{2 0 1 6}$ & \\
\hline Huangana Tayassu pecari & 3 & 2.98 & 3 & $\mathrm{H}=1.72, \mathrm{P}=0.42$ \\
Majas Cuniculus paca & 2.87 & 2.96 & 3 & $\mathrm{H}=5.78, \mathrm{P}=0.05$ \\
Sajino Pecari tajacu & 2.72 & 2.82 & 2.83 & $\mathrm{H}=1.49, \mathrm{P}=0.47$ \\
Venado rojo Mazama americana & 2.24 & 2.27 & 2.57 & $\mathrm{H}=3.78, \mathrm{P}=0.15$ \\
Venado cenizo Mazama nemorivaga & 1.95 & 2.11 & 2.17 & $\mathrm{H}=1.11, \mathrm{P}=0.57$ \\
Consenso o Eigenvalue $>3$ & 6.7 & 6.54 & 9.59 & \\
Acuerdo de cazadores (0 a 1) & 0.69 & 0.66 & 0.73 & \\
$\mathrm{~N}^{\circ}$ de entrevistas & 34 & 33 & 23 & \\
\hline
\end{tabular}




\section{DISCUSIÓN}

Casi todos los modelos de sostenibilidad de la caza fueron congruentes al indicar que el aprovechamiento está dentro de los niveles de sostenibilidad. Es decir, el plan de manejo que incluye la cuota de extracción anual de $300 \mathrm{~kg}$ de carne ahumada por persona para las especies permitidas en la zona de caza conforma una buena estrategia de conservación de los bosques de la Ecorregión Napo. Además, la estrategia de caza de los kichwas, que se orienta al uso de las collpas, hábitats con mayor productividad y las restingas altas contribuye a la sostenibilidad de la caza. El respeto a la norma establecida por los profesionales de la conservación y el uso de una estrategia adecuada implementada por los cazadores aseguran un aprovechamiento sostenible de los animales de caza. Por lo tanto, este primer plan de manejo funcional con fines comerciales en la Amazonía peruana, debe de considerarse como una estrategia adaptable a seguir.

A diferencia de los otros modelos, el modelo de abundancia, que sólo analizó información de la temporada de vaciante, señaló disminución a través del tiempo; tal estimación parece estar relacionada con el incremento gradual de los niveles medios del río. Esto se debe a que las evaluaciones del modelo de abundancia se limitaron a la temporada de vaciante. Los transectos pudieron ser evaluados parcialmente durante la creciente de los ríos y en muchos casos solo se pudieron evaluar finalizando la creciente, siendo estos valores no comparables en el tiempo. Sin embargo, realizar evaluaciones durante la vaciante del río en las mismas zonas por varios años fue muy importante para el análisis de los datos en el tiempo. El modelo de abundancia es afectado por factores naturales (Bodmer \& Robinson, 2004), por ello, este modelo debe ser complementado con información climática o con la fluctuación natural poblacional de las especies (Pérez-Peña, 2007; Fang et al., 2008). La reducción de las estimaciones de la abundancia no necesariamente se debe a la caza, sino también a las crecientes prolongadas del nivel del río.

En la Reserva Nacional Pucacuro, la huangana $T$. pecari es la especie con mayor importancia en la cacería, y su población tiene fluctuaciones naturales cíclicas de una década con causas poco conocidas. Esto podría deberse a causas epidémicas (Fragoso, 2004), mayor producción de individuos machos en poblaciones cercanas a su capacidad de carga (Pérez-Peña, 2007; Fang et al., 2008), ausencia de especies de plantas claves en su alimentación o al incremento anual del nivel del río y/o inundaciones intensas. Para T. pecari el bosque inundable es muy importante (Fang et al., 2008), porque se alimenta de plantas, como la ñejilla Bactris sp. y la huiririma Astrocaryum jauari, que sincronizan su época de fructificación con la temporada de vaciante y crecen en el bosque inundable. Cuando la temporada de creciente se prolonga, abarca el tiempo de fructificación de estas palmeras y la huangana no puede alimentarse, por estar en el bosque inundado (Bodmer et al., 2014).

El tamaño del grupo y la productividad del tipo de hábitat juegan un rol fundamental en los movimientos de esta especie (Carrillo et al., 2002; Pérez \& Flores, 2006); a mayor escasez de alimento es mayor la distancia de movimiento. Durante los meses de junio y julio, hay abundante frutos en el piso de los aguajales, y se pudo observar muchos individuos de huangana durante días o semanas en este hábitat. Si no hay frutos, esta especie busca otro hábitat con gran disponibilidad de alimento. Se tiene que tomar en cuenta todas estas consideraciones al momento de analizar los posibles cambios poblacionales. En lugares donde la huangana es abundante, el sajino es muy escaso y viceversa (Salovaara et al., 2003; Fang et al., 2008), quizá por este motivo el sajino tiene densidades muy bajas en toda la reserva. Las especies de venados y el sajino Pecari tajacu, además de ocurrir en bajas densidades, usualmente tienen poblaciones más estables que la huangana (Pérez-Peña, 2007).

El modelo de abundancia no coincidió con los patrones del modelo de esfuerzo quizá debido a la diferencia del diseño de muestreo y tiempo de colecta de la información. Los análisis del modelo de abundancia usaron información colectada en la época de vaciante mientras que el análisis del modelo de esfuerzo uso información de caza de todo el año aunque los cazadores evitan momentos de extrema inundación porque impide el éxito de cacería (SERNANP, 2013). El modelo de esfuerzo usa información de los cazadores, quienes conocen otras señales para encontrar a sus presas (Pérez Peña et al., 2012). A diferencia de transectos que sólo usan avistamientos y necesitan de grandes esfuerzos o recorridos para estimar las abundancias con mayor precisión (De Thoisy et al., 2008) y pueden subestimar la abundancia de algunas especies, por ejemplo los ungulados (Fragoso et al. 2016). Los resultados de área de ocupación no mostraron reducción drástica como los resultados de avistamientos por transectos, porque además de avistamientos incluye registros indirectos como huellas y olores. Por esta razón nuestros modelos involucran varios métodos de evaluación para contrastarlos entre ellos.

A pesar que el modelo de abundancia indicó que 
las especies son vulnerables debido al incremento del nivel del río y lo prolongado de la inundación crítica, el modelo de cosecha indicó que la caza de las cuatro especies analizadas está por debajo de los límites sostenibles, coincidiendo con el modelo de esfuerzo que no evidenció ninguna disminución poblacional. La caza regulada mediante cuotas de extracción y zonas de caza, ayuda a las especies a permanecer en el lugar. Si dos efectos, como las crecientes prolongadas, que es un indicador de cambio climático (Bodmer et al., 2014) y la sobrecaza, actúan juntos, las disminuciones de los animales serían más drásticas, pudiendo acelerarse más del $50 \%$, pero si actúan independientemente su efecto sería menor (Mora et al., 2006; Carroll, 2007).

El modelo de consenso cultural coincidió con los resultados del modelo de esfuerzo, ambos no evidenciaron disminución poblacional. A pesar de que el modelo de esfuerzo indicó que T. pecarí fluctúa en el tiempo y el de consenso cultural que hay un incremento en la percepción de la abundancia de C. paca, ambos coinciden que no hubo declinación en las poblaciones.

La información de la abundancia, cuotas y sostenibilidad de la caza deben sincronizarse adecuadamente para ayudar a tomar la mejor decisión en la gestión de un área protegida. En un año determinado, la caza puede parecer sostenible, pero si hay algún efecto natural o antrópico que pone en riesgo el aprovechamiento sostenible, las cuotas de cosecha deberían de ajustarse para lograr conservar las poblaciones naturales, por ello, el monitoreo usando varios modelos, es fundamental. Es decir, el monitoreo del plan de manejo de animales de caza debe de usar el modelo de cosecha, abundancia, esfuerzo y consenso cultural como herramientas claves para una gestión proactiva, porque va a permitir detectar los problemas con tiempo suficiente, antes de que sean difíciles de solucionar.

La funcionalidad de este plan es muy importante porque ayuda a la conservación de toda la estructura del bosque, debido a que los animales de caza como los pecaríes, venados y majas ayudan al mantenimiento de la estructura del bosque, por depredación o por dispersión de grandes semillas. La ausencia de estos grandes animales ocasionaría un efecto cascada en el ecosistema terrestre. Actualmente la reserva alberga una alta biodiversidad de vertebrados, con 99 especies de anfibios, 90 de reptiles, 559 de aves y 140 de mamíferos (SERNANP, 2013), y es considerada una de las más biodiversas en la Amazonía peruana, porque alberga el $39.6,45.0,69.4$ y $47.8 \%$ del total de las especies registradas. Por ello, las áreas protegidas con planes de uso sostenible de animales de caza pueden cumplir sus dos grandes objetivos: conservar el ecosistema y beneficiar a los pobladores locales.

Los análisis de sostenibilidad de la caza son claves para iniciar un plan de manejo comercial, pero no es el único aspecto a tomar en cuenta. Un uso sostenible del recurso no es garantía para que los usuarios incrementen sus ingresos económicos (Chirif, 2012), más aún si no tienen autonomía en elegir al mejor comprador. Una preocupación inicial del plan de manejo con fines comerciales, fue poner en riesgo la seguridad alimentaria de los pobladores Kichwas, asumiendo que la cacería que debiera ser consumida en sus pueblos sería vendida a las grandes ciudades. Una serie de entrevistas realizadas a 24 cazadores demostraron que las especies preferidas para la alimentación no son las mismas para la venta, excepto el majas $C$. paca. Los kichwas prefieren animales pequeños para su alimentación, como paujil Mitu salvini, majas C. paca, perdiz Tinamus major, pucacunga Penelope jacquacu y pava Pipile cumanensis, mientras que para la venta prefieren el majas C. paca, huangana $T$. pecari y sajino $P$. tajacu. Si se considera al majas C. paca y la pucacunga P.jacquacu como especies preferidas exclusivamente para la alimentación y son abundantes en los territorios de las comunidades kichwa (Perez-Peña et al., 2012), la venta de los animales que provienen de la reserva no pone en riesgo la seguridad alimenticia de los kichwas.

Uno de los problemas de los cazadores es que dependen económicamente de los comerciantes habilitadores, quienes consideran tener privilegios sobre la carne extraída y a su vez compran la carne a precios muy bajos que no permiten tener ganancias sustanciales, a pesar de ser una carne que proviene de una caza sostenible.

En la actualidad, en la comunidad Kichwa de 28 de Julio, el precio del kilogramo de carne de sajino, huangana, majas o venado oscila entre siete a nueve soles, mientras que en la ciudad de Iquitos esta alrededor de 20 soles (SERNANP, 2014). Algunos restaurantes están dispuestos a pagar hasta 40 soles el kilogramo, aunque la mayoría coinciden en pagar 25 soles (Escobedo et al., 2013). Es decir, si los cazadores redireccionan sus mercado y se capitalizan económicamente, quizá mediante colaboraciones de los miembros del propio grupo de cazadores, pueden sacar más provecho a la caza sostenible. Si se capacitan en la mejora de la presentación de la carne de monte, obteniendo la autorización sanitaria, que mayormente considera normas para carne provenientes de zoocría y no del medio natural, podrían posicionar su actividad en un 
grado de sostenibilidad interesante. Por tal razón, es fundamental el trabajo conjunto de las instituciones involucradas en este tema.

\section{CONCLUSIÓN}

Los diferentes modelos no evidenciaron sobrecaza. El modelo de abundancia que uso información sólo de la temporada de vaciante, indicó vulnerabilidad influenciada por el incremento gradual del nivel del río. Es decir, las normas establecidas en el plan de manejo, como la cuota de extracción anual de $300 \mathrm{~kg}$ de carne ahumada por cazador, caza restringida a las zonas establecidas y sobre las especies menos vulnerables a la sobrecaza, ayudan a tener una caza sostenible y a conservar el ecosistema terrestre de la cuenca del río Pucacuro. Este estudio pone en evidencia que el plan de manejo de animales silvestres es una alternativa económica para los pobladores locales, incrementando sus ingresos y reduciendo otras actividades que podrían causar daño al bosque. Para que la caza sostenible pueda beneficiar más a los cazadores, es necesario un trabajo conjunto con instituciones claves para cortar la dependencia de los comerciantes habilitadores.

\section{AGRADECIMIENTOS}

Este trabajo fue motivado por el conocimiento innato sobre la caza de los kichwas de la comunidad 28 de Julio, quienes aún mantienen vivas sus raíces ancestrales. Un agradecimiento a los guardaparques William Manihuari, Lenner Sandi, Samuel Aguinda, Andy Cariajano, Jorge Ruiz, Jorge Gaviria, Maicon Hidalgo, Italo Celis y Jhancy Segura, por ayudar en las evaluaciones poblacionales. A Lourdes Ruck, Diana Meza, María Riveros, Claudia Ríos, por sus experiencias que fueron claves. A Gloria Rojas, Pedro Mayor, Mark Bowler por las sugerencias en diferentes etapas del plan de manejo. A Jessica Olortegui, Daniel Montes y a todos los estudiantes de los cursos de manejo de fauna silvestre del 2013 y 2015 , por la ayuda en las entrevistas a los cazadores. A todos ellos, nuestros sinceros agradecimientos.

\section{BIBLIOGRAFÍA CITADA}

Altrichter, M.; Taber, A.; Beck, H.; Reyna-Hurtado, R.; Lizarraga, L.; Keuroghlian, A.; Sanderson, E.W. 2012. Range-wide declines of a key Neotropical ecosystem architect, the Near Threatened white-lipped peccary Tayassu pecari. Oryx, 46(1): 87-98.

Aquino, R.; Gil, D.; Pezo, E. 2009. Aspectos ecológicos y sostenibilidad de la caza del majás
(Cuniculus paca) en la cuenca del río Itaya, Amazonía peruana. Revista Peruana de Biología, 16(1): 67-72.

Ayres, M.; Ayres Jr, M.; Lima-Ayres, D.; Dos Santos, A. 2007. Bioestat. Aplicacioes estatisticas nas áreas das ciencias bio-medicas. Sociedade Civil Mamirauá, MCT-CNPq, Belem do Para, 324pp.

Beck-King, H.; Von Helversen O.; Beck-King, R. 1999. Home range, population density, and food resources of Agouti paca (Rodentia: Agoutidae) in Costa Rica: A Study using alternative methods. Biotropica. 31(4): 675-685.

Bello, C.; Galetti M.; Pizo M.A.; Magnago L.F.S.; Rocha M.; Lima R.A.F.; Peres C.A.; Ovaskainen O.; Jordano P. 2015. Defaunation affects carbon storage in tropical forests. Science Advances: 110

Bendayán-Acosta, N.Y.; Bardales-García, J.; VerdiOlivares, L. 2004. Catastro de zonas de extracción de fauna silvestre en Loreto, Perú. MEMORIAS: Manejo de Fauna silvestre en Amazonia y Latinoamérica. p. 499-503.

Biondo, C.; Keroghlian, A.; Gongora, J.; Miyaki, C. 2011. Population genetic structure and dispersal in white-lipped peccaries (Tayassu pecari) from the Brazilian Pantanal. Journal of Mammalogy, 92(2): 267-274.

Bodmer R.E.; Fang T.G.; Puertas P.E.; Antúnez M.; Chota K.; Bodmer W. 2014. Cambio climático y fauna silvestre en la Amazonía peruana. Impacto de la sequía e inundaciones intensas en la Reserva Nacional Pacaya Samiria. Wust Ediciones. Fundamazonia. Iquitos. 254 pp.

Bodmer, R.; Robinson, J. 2004. Evaluating the Sustainability of Hunting in the Neotropics. In: Silvius, K.; Bodmer, R.E.; Fragoso, J.M.V. (Eds). People in Nature. Wildlife conservation in south and central America. p. 199-323.

Bodmer, R.; Eisenberg, J.; Redford, K. 1997. Hunting and the likelihood of extinction of Amazonian mammals. Conservation Biology, 11 (2): 460-466.

Borgatti, S.P.; Everett, M.G.; Freeman, L.C. 2002. Ucinet for Windows: Software for social network analysis. Harvard, MA: Analytic Technologie.

Bowen-Jones, E. 2003. Bushmeat: traditional regulation or adaptation to market forces. In: Olfield S. (Ed.). The trade in wildlife: Regulation for conservation. p. 132-145.

Burnham, K.; Anderson, D.; Laake, J. 1980. Estimation of density from line transect sampling of biological populations. Wildlife Monographs, 72:3-202. 
Carrillo, E.; Saenz, J.; Fuller, T. 2002. Movements and activities of white lipped peccaries in Corcovado National park, Costa Rica. Biological Conservation, 108: 317-324.

Carroll, C. 2007. Interacting effects of climate change, landscape conversion, and harvest on carnivore populations at the range margin: Marten and Lynx in the Northern Appalachians. Conservation Biology, 21(4): 1092-1104

Chirif, A. 2012. La Historia del Tahuayo contada por sus moradores. Wildlife Conservation Society, Lima. 99pp.

Davies, G. y D. Brown. 2007. Bushmeat and Livelihoods: Wildlife Management and Poverty Reduction. Conservation Science and Practice Series. Blackwell Publishing, London. 274pp.

De Thoisy, B.; Brosse, S.; Dubois, M.A. 2008. Assessment of large-vertebrate species richness and relative abundance in Neotropical forest using line-transect censuses: what is the minimal effort required?. Biodiversity Conservation, 17: 2627-2644.

Escobedo-Grández, A.J.; Pérez Peña, P.E.; Saldaña, H.C.; Burga, R.L. 2013. Consumo de la carne de monte o "carne invisible" en restaurantes turísticos de Iquitos. Reporte Técnico. Reserva Nacional Pucacuro-SERNANP, Iquitos. 20 pp.

Fang, T.; Bodmer, R.E.; Puertas, P.; Mayor, P.; Pérez-Peña, P.E.; Acero, R.; Haymann, D. 2008. Certificación de pieles de pecaries en la Amazonía peruana: Una estrategia para la Conservación y Manejo de Fauna en la Amazonía Peruana. Wust Ediciones, Lima. $203 \mathrm{pp}$

Fragoso, J.M.V.; Levi T.; Oliveira L.F.B.; Luzar J.B.; Overman H.; Read J.M.; Silvius K. 2016. Line Transect Surveys Underdetect Terrestrial Mammals: Implications for the Sustainability of Subsistence Hunting. PlosOne. 11(4): 1-18.

Fragoso, J.M.V. 2004. A long - term study of whitelipped peccary (Tayassu pecari) population fluctuation in northern Amazonia. Anthropogenic vs "natural" causes. In: K. Silvius, R. Bodmer, and J.M.V Fragoso (Eds). People in Nature. Wildlife conservation in south and central America. p. 286-296.

Ganoza-Durant, C; Stiglich-Watson, A. 2015. El Perú está calato. El falso milagro de la economía peruana y las trampas que amenazan nuestro progreso. Ed. Planeta, Lima. 161pp.

Harrison, R.D.; Tan, S.; Plotkin, J.B.; Slik F.; Detto, M.; Brenes, T.; Itoh, A.; Davies, S.J. 2013. Consequences of defaunation for a tropical tree community. Ecology Letters: 1-8.
Higgins, M.A.; Ruokolainen, K,; Tuomisto, H,; Llerena, N,; Cardenas ,G,; Phillips O,; Vasquez R.; Rasanen, M. 2011. Geological control of floristic composition in Amazonian forest. Journal of Biogeography, 38: 2136-2149

Keuroghlian A.; Eaton D.P.; Longland W. 2004. Area use by white-lipped and collared peccaries (Tayassu pecari and Tayassu tajacu) in a tropical forest fragment. Biological Conservation. 120: 411-425

Moya, K. E. 2011. Monitoreo de la comercialización de carne de monte en los mercados de Iquitos y estrategias para su conservación. Tesis de pregrado, Universidad Nacional de la Amazonia Peruana, Facultad de Ciencias Biológicas, Iquitos, Perú. 73pp.

Mayor, P.; Guimarães, D.A.; Le Pendu, J.V.; da Silva, F.; Jori, F.; López-Béjar, M. 2007. Reproductive performance of captive collared peccaries (Tayassu tajacu) in the eastern Amazon. Animal Reproduction Science, 102(12): 88-97.

Mayor, P.; Bodmer R.R.; Lopez-Bejar, M. 2010. Reproductive performance of the wild whitelipped peccary (Tayassu pecari) female in the Peruvian Amazon. European Journal of Wildlife Research, 55: 631-634.

Mayor, P.; Bodmer,R.E.; López-Béjar, M.; LópezPlana, C. 2011. Reproductive biology of the wild red brocket deer (Mazama americana) female in the Peruvian Amazon. Animal Reproduction Science, 128: 123-128

Mora C.; Metzger R.; Rollo A.; Myers R. 2007. Experimental simulations about the effects of overexploitation and habitat fragmentation on populations facing environmental warming. Proceedings of the Royal Society B: 274(1613).

Osuri A.M.; Ratnam J.; Varma V.; Alvarez-Loayza P.; Hurtado Astaiza J.; Bradford M.; Fletcher C.; Ndoundou-Hockemba M.; Jansen P.A, Kenfack D. 2016. Contrasting effects of defaunation on aboveground carbon storage across the global tropics. Nature Communications. 7:11351

Pérez, P.E.; Flores, J. 2006. Fluctuación estacional de mamíferos y aves en la concesión de conservación de lago Preto-Paredón: reporte técnico. WCS \& DICE, Iquitos. 47pp.

Pérez-Peña, P.E. 2007. Refining the guidlines of the peccary pelt certification programme. Tesis de Maestría, Universidad de Kent, Durrell Institute of Conservation and Ecology, Canterbury, Reino Unido. 75pp.

Pérez-Peña, P.E.; Ruck, L.; Riveros, M.S.; Rojas, G. 2012. Evaluación del conocimiento indígena 
Kichwa como herramienta de monitoreo en la abundancia de animales de caza. Folia Amazónica, 21(1-2): 115-127.

Pulgar Vidal, M.; Monteferri, B.; Dammert, J.L. 2010. The trade-offs between conservation and extractive industries. In: N. Leader-Williams, W.M. Adams y R.J. Smith (Eds.). Trade-Offs in Conservation: Deciding What to Save. p. 233252.

Salovaara, K.; Bodmer, R. M. Recharte and C. Reyes. 2003. Diversidad y Abundancia de Mamíferos. In: N. Pitman, C. Vriesendorp y D. Moskovits (Eds.). Perú: Yavarí. Rapid Biological Inventories 11. p. 74-84.

SERNANP. 2013. Reserva Nacional Pucacuro: Diagnóstico del proceso de Elaboración del Plan Maestro 2013-2018. Servicio Nacional de Áreas Naturales Protegidas por el Estado, Lima. 106pp.

SERNANP. 2014. Plan de manejo de animales de caza en la Reserva Nacional Pucacuro. Realizado por cazadores kichwas de la comunidad 28 de julio, Alfonso Ugarte y Asociación de Cazadores de Intuto. Servicio Nacional de Áreas Naturales Protegidas por el
Estado, Lima. 38pp.

Swamy V.; Terborgh J.T.; Álvarez-Loayza P.C.; Cornejo-Valverde F.; LaTorre Farfán J.P.; Vela C.I.A.; Chillihuani. Coronado J.J. 2013. El impacto de desfaunación sobre la regeneración del bosque en la cuenca del río Madre de Dios: resultados preliminares de un estudio de largo plazo. Reporte Manu 2013. In: Groenendijk, Tovar, Wust (Eds). Pasion por la Investigación en la Amazonía peruana. p. 138-153.

Van Holt, T.; Towsend, W. R.; Cronkleton, P. 2010. Assessing local knowledge of game abundance and persistence of hunting livelihoods in the Bolivian Amazon using consensus analysis. Human Ecology, 38: 791-801.

Van Vliet, N. 2011. Livelihood alternatives for the unsustainable use of bushmeat. Report prepared for the CBD Bushmeat Liaison Group. Technical Serices, 60. SCBD, Montreal, 46pp.

Weller, S.C. 2007. Cultural consensus theory: applications and frequently asked questions. Field Methods, 19:339-368.

Recibido: 11 de Enero del 2016

Aceptado para publicación: 15 de Febrero del 2016 